\title{
Behavioral and histopathological studies of cervical spinal cord contusion injury in rats caused by an adapted weight-drop device
}

Kanyaratana Bamrungsuk', Anchalee Vattarakorn', Namphung Thongta', Kanokwan Tilokskulchai', Sompol Tapechum', Supin Chompoopong 2,*

Abstract

Background: Models of spinal cord injury (SCI) caused by weight-drop devices to cause contusion have been used extensively, and transient behavioral deficits after thoracic injury have been demonstrated. The severity of the injury caused by the device should be mild enough to allow recovery.

Objective: To determine whether our adapted weight-drop device with a small tip can effectively induce mild hemicontusion at the level of the fifth cervical vertebra.

Methods: We divided 15 adult male Sprague Dawley rats into groups of 5 for the following treatments: sham (SH, laminectomy only), mild (MSCI) or severe SCI (SSCI). Behavioral tests and histopathology were used before (day 1) and after the treatment on days $3,7,14,21,28$, and 35 to assess the injury.

Results: Rats with SSCI showed a significant somatosensory deficit on days 3 and 7 compared with rats in the SH group, recovering by day 14. In a horizontal-ladder test of skilled locomotion, rats with SSCI showed a significant increase in error scores and percentage of total rungs used, and a decrease in the percentage of correct paw placement compared with rats in the SH group. There was greater recovery to normal paw placement by rats with MSCI than by rats with SSCI. These behavioral deficits were consistent with histopathology using hematoxylin and eosin counterstained Luxol fast blue, indicating the degree of injury and lesion area.

Conclusions: Mild hemicontusion caused by the adapted device can be used to evaluate SCI and provides a model with which to test the efficacy of translational therapies for SCI.

Keywords: animal experimentation; contusions; motor activity; motor skills; spinal cord injuries

Animal models of spinal cord injury (SCI) using contusion have been developed to mimic signs and symptoms similar to those seen after SCI in humans [1] because most SCIs in humans involve spinal cord contusion. Histopathology in these models show an intact dura and external aspects of the spinal cord, but an internal cystic cavity. The loss of motor

*Correspondence to: Supin Chompoopong, Department of Anatomy, Faculty of Medicine, Siriraj Hospital, Bangkok 10700, Thailand, e-mail: supin.cho@mahidol.ac.th

'Department of Physiology, Faculty of Medicine, Siriraj Hospital, Mahidol University, Bangkok 10700, Thailand

${ }^{2}$ Department of Anatomy, Faculty of Medicine, Siriraj Hospital, Mahidol University, Bangkok 10700, Thailand

¿ Open Access. ๑ 2018 Kanyaratana Bamrungsuk et al., published by Sciendo. (cc) BY-NC-ND This work is licensed under the Creative Commons Attribution NonCommercial-NoDerivatives 4.0 License. 
and sensory function correlates with the preservation of white and gray matter [2]. SCI causes cellular damage in the gray matter, and the loss of the functional ascending and descending axonal signals in the white matter depends on the severity of the lesion.

In contusion injury of the fifth cervical vertebra (C5), rats show clubbing of their forepaws and perturbed grasping ability [2]. A horizontal-ladder test is sufficiently sensitive enough to study forelimb functional deficits resulting from cervical injury, forelimb compensation, adaptation, and recovery [3]. In addition to loss of motor function, SCI can also cause loss of sensation or hypersensitivity to stimuli, and symptoms of pain [4]. To examine the somatosensory asymmetry after hemicontusion injury, a bilateral tactile stimulation test called the adhesive sticker removal task has been used [5].

The successful evaluation of any therapeutic regimen for SCI depends on the reliability and reproducibility of experimental animal models of SCI. The severity of the injury should be mild enough to allow recovery. However, to our knowledge, there have been no appropriate models to date, with which to study the behavioral consequences, anatomical changes, and susceptibility to a mild contusion at a cervical level. The initial mechanical disruption of tissue depends on the impact force delivered to the spinal cord and leads subsequently to complex histopathological changes and neurological impairment. In the present study, we used a weight-drop device adapted with a tip diameter smaller than that of commercial devices to induce a hemicontusion injury at C5 and generate a precisely located SCI lesion in rats. To evaluate the efficacy of the adapted device, a fixed weight was used and dropped from various heights, followed by behavioral tests and histopathology.

\section{Materials and methods}

Male Sprague Dawley rats weighing 180-200 g were obtained from the National Laboratory Animal Center, Mahidol University, Nakhon Pathom, Thailand. The rats were housed in flat-bottom plastic cages with standard laboratory rat food and water available ad libitum and under a standard 12-h lightdark cycle, at $25 \pm 1^{\circ} \mathrm{C}$. Experiments were performed after the rats had been acclimated to their new environment in our laboratories for at least 1 week. All procedures followed the Institute of Laboratory Animal Resources, Commission on Life Sciences, National Research Council "Guide for the Care and Use of Laboratory Animals" Washington, D.C.: National Academy Press; 1996 and were approved by Animals Experiments Ethics Committee of Mahidol University (approval No. 013/2555).

\section{Adapted weight-drop device}

The weight-drop device for SCI was adapted from that described by Soblosky et al. [3] (Figure 1A). It consisted of an $8 \mathrm{~cm}$ long metal rod with $13.22 \mathrm{~g}$ total weight and a $1.45 \mathrm{~mm}$ tip diameter (Figure 1B), which is less than the diameter $(2.2-2.5 \mathrm{~mm})$ of a commercial spinal impactor injury device.

The sterilized impactor rod was inserted into a plastic tube that was made from a $10 \mathrm{~mL}$ disposable pipette cut off at both ends. The rod fitted loosely and slid freely through the pipette at the lower end with minimal friction and without lateral movement. The upper part of the rod was held by a string attaching to a pulley. The tip of rod was at first placed gently on the upper surface of dura mater, and the rod was raised by the string to various heights ( 4 or $6 \mathrm{~mm}$ ), and then the pulley was locked. The pulley was then unlocked to allow the rod to drop and contuse the cord, generating a SCI (Figure 1C and D), and then the rod was raised again immediately. The weight of the rod and the various heights were recorded to calculate the final force of contusion, for $4 \mathrm{~mm}$ height, $5.18 \times 10^{-4} \mathrm{~N}$ as force for MSCI and for $6 \mathrm{~mm}$ height, $7.77 \times 10^{-4} \mathrm{~N}$ as force for SSCI.

\section{Surgical procedure}

Surgery was performed under anesthesia using a mixture of ketamine $(120 \mathrm{mg} / \mathrm{kg})$ and xylazine $(8.5 \mathrm{mg} / \mathrm{kg})$. Using sterile technique throughout the surgery, the neck region was shaved and prepared aseptically for a midline dorsal skin incision that was made between the spinous processes of the $\mathrm{C} 2$ and $\mathrm{C} 7$ vertebrae; the trapezius was incised at the midline; and the underling paravertebral muscles at $\mathrm{C} 4-\mathrm{C} 6$ were resected to expose the spinal lamina. A bilateral laminectomy was performed at C5 to expose the dorsal aspect of the spinal cord. The spinal column was stabilized by rigidly clamping the vertebral body of $\mathrm{C} 2$ and the spinous process of the $\mathrm{C} 7$ vertebra with toothed forceps that were connected to the supporting arms of an instrument stand. The right side of the C5 spinal cord was contused with the tip of the metal rod that brought over the laminectomy site and set perpendicular to the surgical table (Figure 1E).

\section{Postoperative care}

Immediately after surgery the rats were kept under a heating lamp until they regained consciousness. For 5 days, the rats were received subcutaneous injections daily of 5\% dextrose and $0.45 \%$ sodium chloride injection $(5 \mathrm{~mL})$, ampicillin 

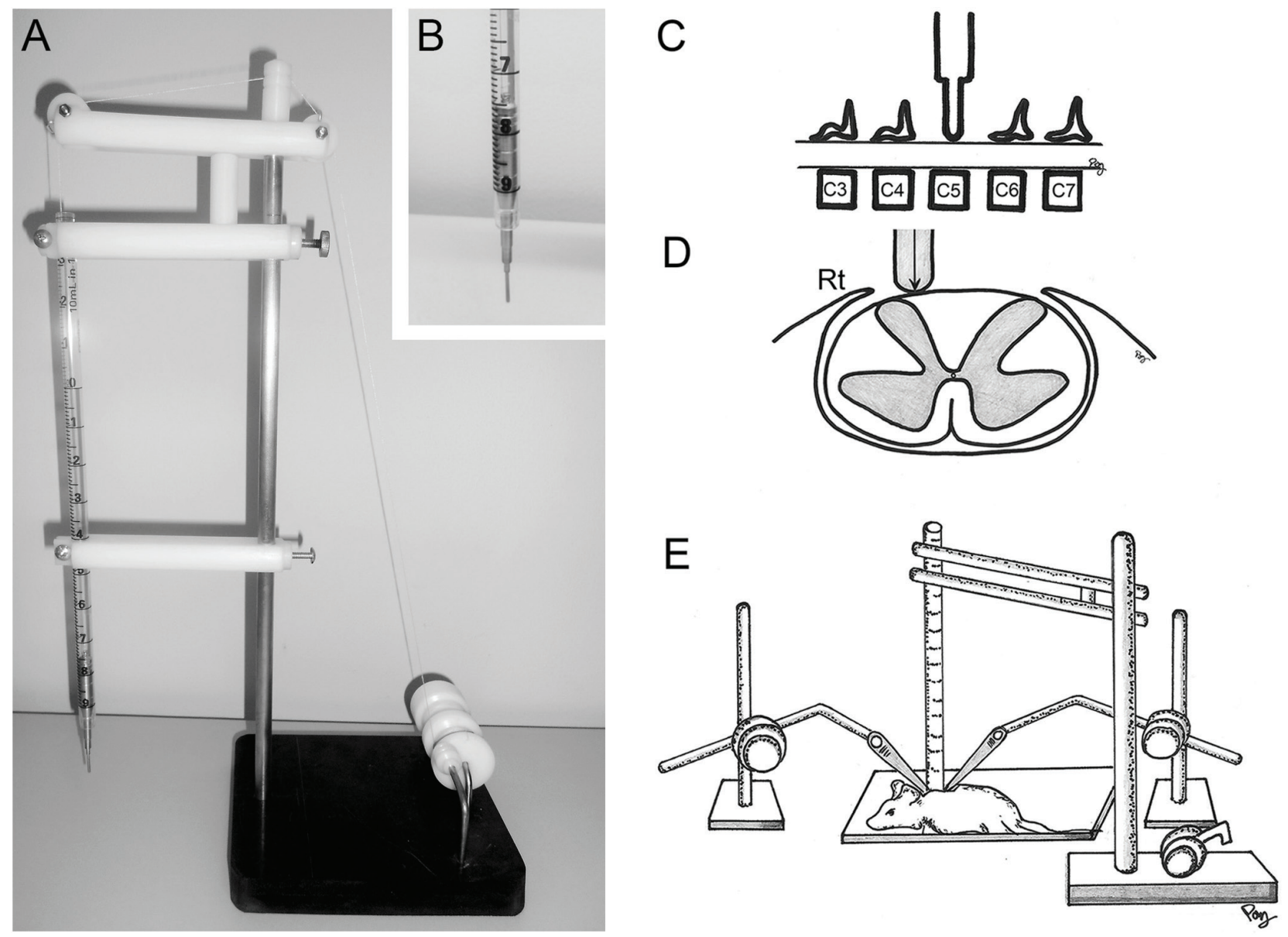

Figure 1. Adapted weight-drop device used to cause hemicontusion spinal cord injury (A, B). The metal rod was applied to the right side (Rt) of the $\mathrm{C} 5$ spinal cord (C, D). The spinal column was stabilized by rigidly clamping the vertebral body at $\mathrm{C} 2$ and the spinous process of the $\mathrm{C} 7$ vertebra with toothed forceps that were connected to supporting arms of an instrument stand (E)

(20 mg/kg), and tramadol ( $5 \mathrm{mg} / \mathrm{kg}$ ). The rats were observed to ensure that they were able to eat and drink without assistance. Feed pellets were placed directly on the bedding, individual water bottles with sippers placed just above the level of the bedding. To stimulate appetites after surgery, high glucose cereals were offered. Their bladder function was assessed and the rats observed for micturition to ensure that there were no urination problems. Manual expression was used initially as necessary.

\section{Animals}

We allocated 15 adult male Sprague Dawley rats to 3 groups of 5 rats that would undergo the following treatments: laminectomy only or sham (SH), mild spinal cord injury (MSCI), or severe spinal cord injury (SSCI). To induce SCI, the metal rod was held at a $4 \mathrm{~mm}$ from the spinal cord for MSCI or
$6 \mathrm{~mm}$ height for SSCI. The rats in the SH (laminectomy only) group served as controls. The rats were maintained for measurements for 5 weeks.

\section{Adhesive paper removal task}

Rats were acclimatized to their environment and tested for somatosensory function. Two small pieces of self-adhesive paper (stickers, equal size, about $10 \mathrm{~mm} \times 10 \mathrm{~mm}$ ) were applied to the dorsum of each forepaw innervated by the $\mathrm{C} 5$ dermatome [6]. Adult rats normally touched and removed stickers with their teeth. The time to remove a sticker was recorded for each forepaw, and the latency time from initial contact to removal was calculated. The time limit of the trial to remove the stickers was $120 \mathrm{~s}$. Each animal underwent 3 trials. If a sticker fell off, the trial was repeated. Trials without either contact or removal within 2 min were recorded as lasting $121 \mathrm{~s}$ 
$[5,7]$. The adhesive sticker removal task was evaluated at 1 day before injury (day 1) and postinjury on days 3, 7, 14, 21, 28 , and 35 .

\section{Horizontal ladder test}

A horizontal ladder test was used to assess skilled locomotion. The horizontal wooden ladder used was $129.39 \mathrm{~cm}$ long and $16.51 \mathrm{~cm}$ wide, and 37 rungs ( $0.79 \mathrm{~cm}$ diameter) were inserted $2.5,3.2$, or $5.7 \mathrm{~cm}$ apart [3]. While rats crossing the ladder, was focused behavior on the side of the injury. The pattern of rungs was changed weekly to prevent animals from learning and adapting to the rung pattern.

Before injury, animals were allowed to explore the test apparatus freely to habituate for 10-20 min on the first day and tested preoperatively to establish a baseline (day 1) and postinjury on days $3,7,14,21,28$, and 35 . Rats were pretrained to traverse the horizontal ladder. Training comprised 3 trials per day for 2 days and then 1 trial a day until the test criterion was achieved. The test criterion was set at an ability to cross the ladder with no more than 1 forepaw misplacement for 5 consecutive trials. Rats were subsequently injured 3-5 days after reaching criterion and did not receive further training during this time [8].

The side of the rats ipsilateral to the injury was recorded with a Logitech Webcam Pro 9000 at 65 images per second to determine the type and number of forepaw placements that were counted by an evaluator who was blinded to the experimental group. The forepaw placements were analyzed and categorized as follows: correct placement, touch, slip, or miss (Figure 2A). For error scoring, a correct placement (score = 0 ) was defined as weight-supported placement of the paw on a

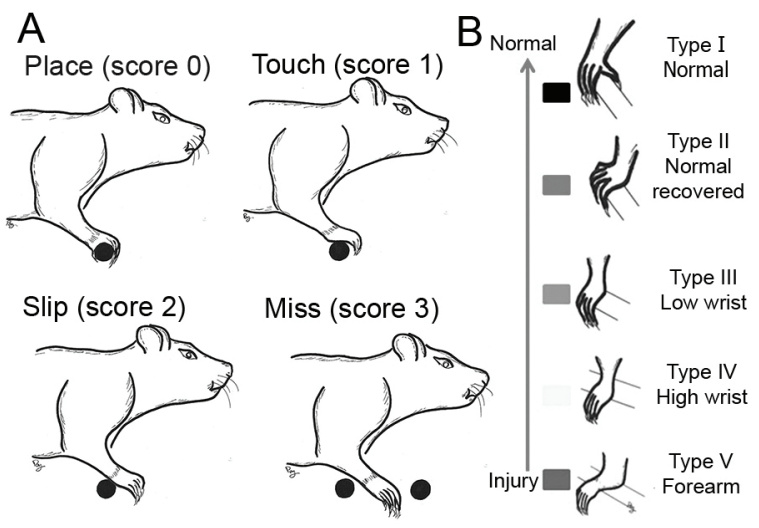

Figure 2. Forepaw placements were categorized as follows: correct placement, touch, slip, or miss (A). Five types of forepaw placement were recorded when the rats attempted to place the palm of their paw directly onto the rung (B) rung with subsequent lift-off. A touch ( as the placement of the paw with no apparent weight support. A slip (score $=2$ ) was defined as the initial contact with the rung and subsequent movement of the wrist of forepaw above the level of the rung. A miss (score = 3) was defined as the movement of the wrist of the forepaw below the level of the rung without initial paw contact. Scores from 3 runs per day were averaged independently for the forepaw of each animal and were referred as the error score. In addition, the percentage of total rungs used ([No. correct + touch + slip + miss]/ No. total rungs $\times 100)$ and the percentage of correct placements $([$ No. correct $/\{$ No. correct $+\operatorname{slip}+\operatorname{miss}\}] \times 100)$ were analyzed [9].

The type of forepaw placement was recorded including whether the rats failed to place the palm of their paw directly onto the rung (Figure 2B). Type I: normal placement consisted of 2 nd -4 th digits placed in front of the rung and the 5th digit behind the rung. Type II: normal recovered placement was the placement after injury in which the paw and palm placement was correct, but 2 nd -5 th digits were in front of the rung. Types III and IV: when the rats did not use their digits to grasp the rungs and usually placed either their lower (type III) or upper wrists (type IV) on the rung for support. Type V: forearm placement, rats used their upper wrist or placed their forearm on the rungs for support [3].

\section{Tissue preparation}

On day 35, rats of all groups were deeply anesthetized with an intramuscular injection of $120 \mathrm{mg} / \mathrm{kg}$ of ketamine combined with xylazine $8.5 \mathrm{mg} / \mathrm{kg}$. Each rat was perfused transcardially with $500 \mathrm{~mL}$ of saline solution, then followed by $500 \mathrm{~mL}$ of $4 \%$ paraformaldehyde solution. After perfusion, 3 segments ( $1 \mathrm{~cm}$ each) of the spinal cord were removed and indicated as lesion segment, rostral penumbra, and caudal penumbra [10]. The segments were fixed in $4 \%$ paraformaldehyde overnight and then dehydrated in a graded series of ethanol solutions and embedded in paraffin.

\section{Histopathology}

Transverse sections of the lesion segment were cut at $16 \mu \mathrm{m}$ thickness [10]. Using hematoxylin and eosin (HE) and Luxol fast blue (LFB) staining to characterize the spinal tissue damage after injury, after deparaffinization, sections were incubated overnight at $60^{\circ} \mathrm{C}$ with $0.1 \%$ LFB in acidified $95 \%$ ethanol, then the color developed with $0.05 \% \mathrm{Li}_{2} \mathrm{CO}_{3}$, and 
counterstained with HE for 5 min. Stained sections were examined under a light microscope at 5-40× magnification.

\section{Statistical analysis}

Differences between groups were compared using a one-way ANOVA followed by a Tukey post hoc test for multiple comparisons or using independent sample $t$ test if only 2 groups were applied. We assumed the data were distributed normally. For $t$ tests involving 1 degree of freedom, a directional test was conducted. Differences were considered significant when $P<0.05$. Results are expressed as mean \pm standard error of the mean (SEM).

\section{Results}

\section{General features}

After injury, all SCI rats showed the paralysis of digits known as "clubbing" on the ipsilateral side and a normal forepaw on the contralateral side (Figure 3). There was no apparent respiratory impairment, bladder dysfunction, or defecation problem. The rats with SCI showed a stress response to surgery including a decrease in food and water intake and decrease in body weight (data not shown). These stress responses were resolved within 3 days of the injury.

\section{Adhesive sticker removal task}

For the adhesive paper (sticker) removal task, on 1 day before surgery (day 1) all rats used the least amount of time to remove sticker (Figure 4). In the sham group, the time increased at day $3(15.00 \pm 2.83 \mathrm{~s})$ and decreased to an average time 4.8 $\pm 5.5 \mathrm{~s}$ until the end of the experiment. Rats with MSCI and
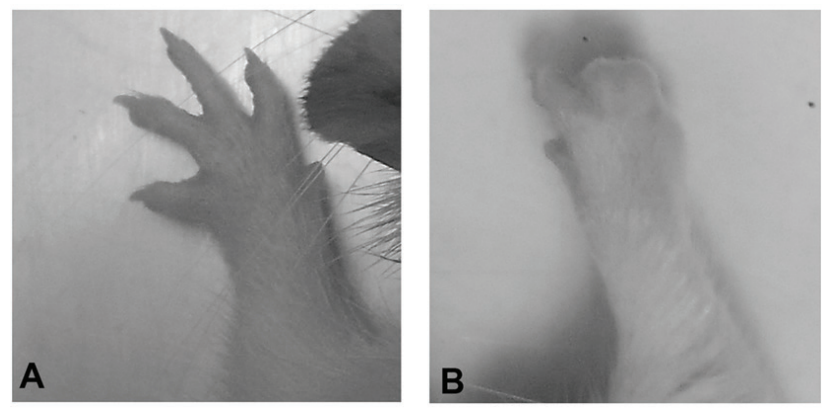

Figure 3. Normal forepaw on the contralateral side of the Sprague Dawley rats (A) and clubbed forepaw on the ipsilateral side (B) after right side hemicontusion of the spinal cord at the level of the $\mathrm{C} 5$ vertebra
SSCI showed a significant somatosensory deficit only on days 3 (MSCI, $P=0.05$; SSCI, $P=0.001$ ) and 7, (MSCI, $P=0.003$; SSCI, $P=0.001$ ), but not after day 14 for MSCI or SSCI when compared with day $1, P<0.05$. Only rats with SSCI showed a significant somatosensory deficit on days 3 and 7 when compared with the SH group, $P<0.05$.

\section{Horizontal ladder test}

For the horizontal ladder test, the characteristics of forepaw placements on rungs are presented (Figure 5A-C). This test was used to analyze the error scores (Figure 5D), the percentage of the total rungs used (Figure $\mathbf{5 E}$ ), and the percentage of correct paw placements (Figure 5F).

On day 1 (the day before treatment), the error scores (Figure 5D) of all groups were approximately 0.67-2.78 points. In the SH group, the error scores were approximately 0.17-3.33 points for all measurements. The error scores of rats with MSCI and SSCI were increased significantly at day 3 and decreased at day 7 when compared with the SH group, $P<0.05$.

The percentage of the total rungs used (Figure 5E), on day 1 of the experiment, was approximately $31 \%-38 \%$ for all groups. In the SH group, the percentage tended to increase on day $3(42 \%)$ and decrease on day 14 (32\%). The percentage of the total rungs used by rats with SSCI was higher than that for rats with MSCI. In the rats with SSCI and MSCI, the

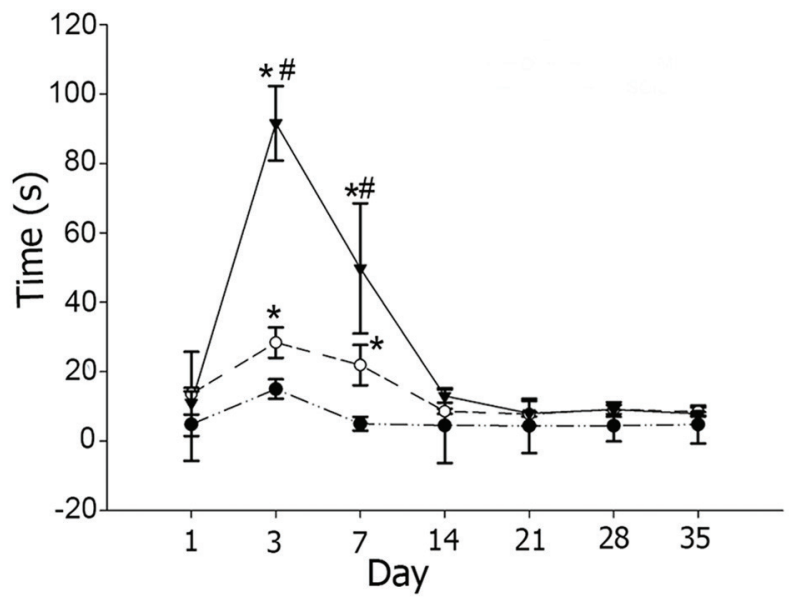

Figure 4. Comparison of adhesive paper (sticker) removal time. For the rats with $\mathrm{MSCl}$ (open circles, dashed line) and SSCIS (inverted solid triangles, unbroken line), the time for removing the sticker was increased and there was a significant somatosensory deficit only on days 3 and 7, but not after day 14 when compared with rats in the sham (laminectomy only) group (solid circles, dashed and dotted line). ${ }^{*} P<0.05$ when compared to day $1 .{ }^{*} P<0.05$ when compared with the $\mathrm{SH}$ group. Error bars indicate standard error of the mean 

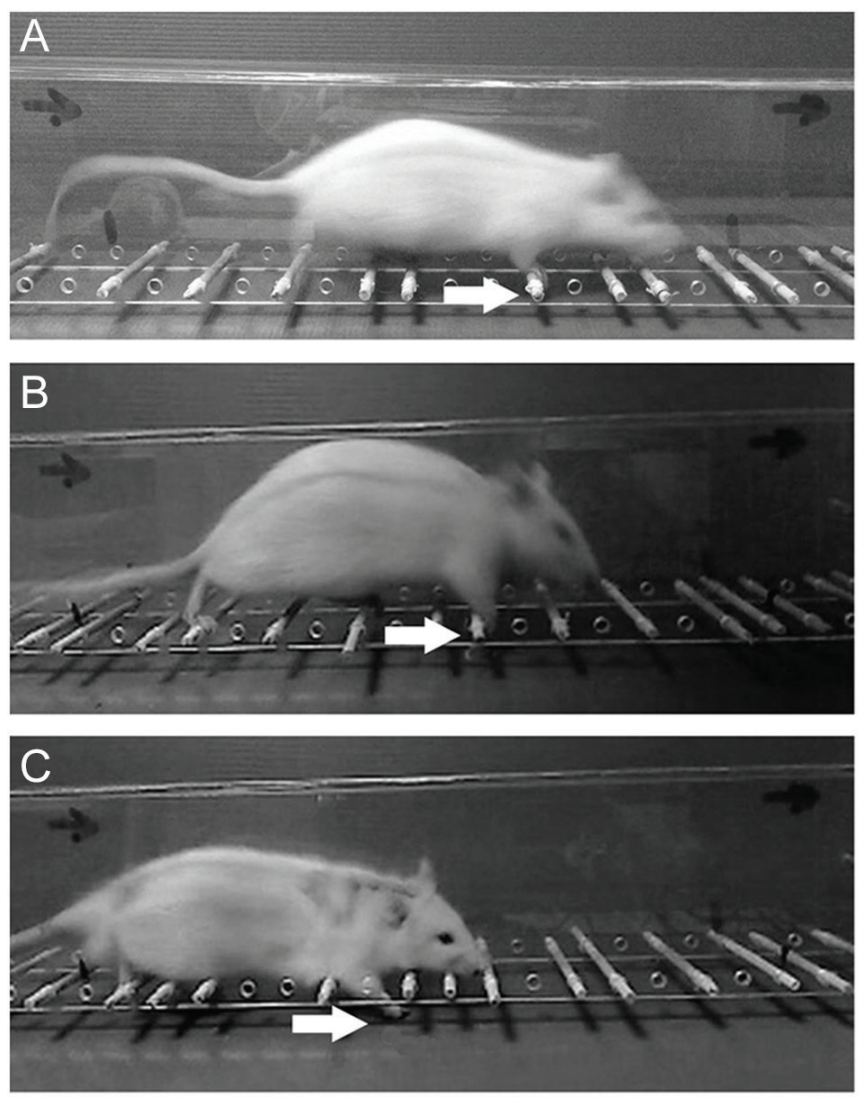

Figure 5. Effect of injury severity on skilled locomotion. The forepaw placement (arrows) is presented as correct placement (A), slip (B), and miss (C). The error scored (D) and the percentage of total rungs used (E) increased significantly on day 3 in rats with $\mathrm{MSCl}$ (open circles, dashed lines) and SSCI (inverted solid triangles, dashed and dotted lines). Rats with $\mathrm{SSCl}$ showed a significantly higher error and number of rungs used on all days tested compared with rats in the SH group (solid circles, unbroken lines). The percentage of correct forelimb placement (F) by rats with $\mathrm{MSCl}$ and $\mathrm{SSCl}$ decreased significantly on day 3. Rats with $\mathrm{SSCl}$ showed significantly less correct placement than rats with $\mathrm{MSCl}$ or $\mathrm{SH}$. ${ }^{*} P<0.05$ when compared with day $1 .{ }^{\#} P<0.05$ compared with rats in the $\mathrm{SH}$ group. Error bars indicate standard error of the mean

percentage was increased significantly on day 3 when compared with rats in the SH group, $P<0.05$. In this study, uneven rung spacing patterns were used, which required precise adjustment of forepaw position. The deficits in rung placing or grasping may result a decrease in the percentage of the total rungs used.

The percentage of the correct placement (Figure 5F), on day 1 of the experiment, was approximately $66 \%-96 \%$ for all groups. Rats in the sham group placed paws correctly in approximately $80 \%-100 \%$ of all experiments. Rats with SSCI placed paws correctly less often than those with MSCI. In the
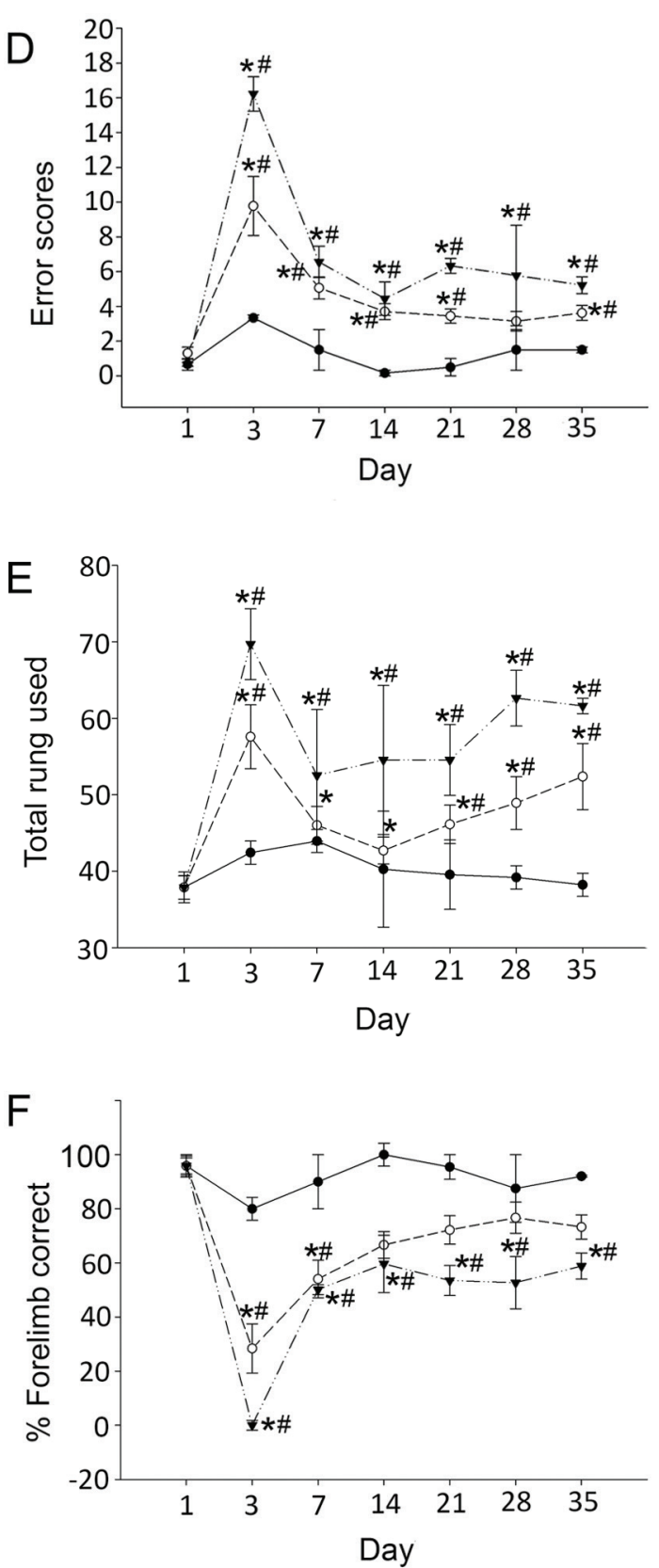

rats with SSCIS and MSCIM, the percentage of correct paw placements decreased significantly on day 3 when compared with rats in the SH group, $P<0.05$.

The type of forepaw placement was used to analyze skilled locomotor function (Figure 6). On day 1 rats in all groups and on all days rats in the SH group used $100 \%$ normal Type 1 placement. After MSCI rats showed not more than $50 \%$ of normal recovery placement (Type II) on day 7. Rats with SSCI showed an average $27 \%$ of forearm placement (Type V) starting at day 3. Normal recovered placement was higher in rats with MSCI than it was in rats with SSCI. 

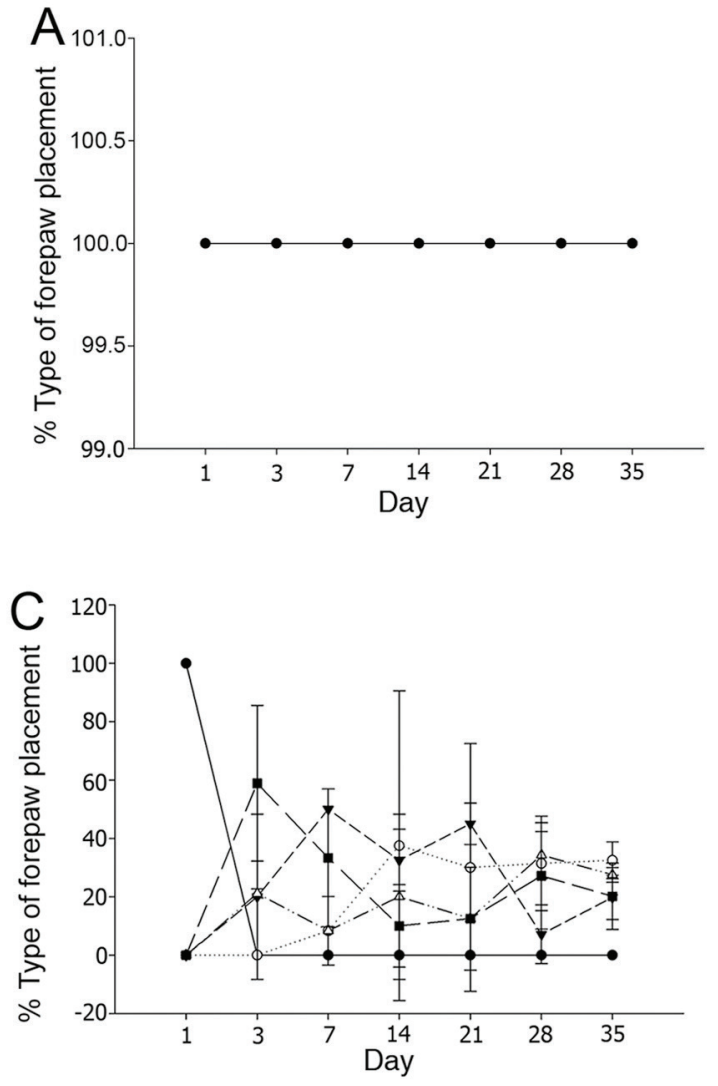

\section{Histopathology}

The adapted weight-drop device produced graded hemicontusion injury as shown by the various sizes and the site of the cystic cavity on the ipsilateral side of the spinal cord at C5 (Figure 7). Using HE counterstained with LFB, the histopathological changes of neuronal tissues were shown in dorsal horn, white matter, and ventral horn of rats in the SH, MSCI, and SSCI groups.

MSCI rats showed the intact dura and external surface of spinal tissue. The cystic cavity was shown in the ipsilateral side of the spinal cord. The cystic cavity was located in dorsolateral funiculus.

SSCI also showed the intact dura mater and external surface of spinal tissue, but a larger ipsilateral cystic cavity located in the dorsolateral funiculus, ventral lateral funiculus, and gray matter of the spinal cord.

\section{Discussion}

Contusion caused by a dropped weight in models of SCI has been used extensively, and suggests the efficacy of mild

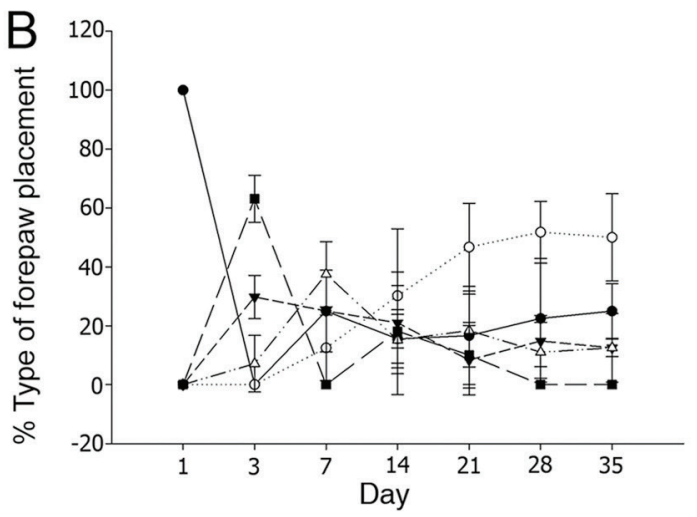

Figure 6. Distributions of types of forepaw placement expressed as percentage on each day. Rats in the sham injury group (laminectomy only) placed their paws $100 \%$ normally or Type I (solid circles, unbroken line) on all days of the experiment (A). Rats with mild spinal cord injury (MSCl) showed recovered placement or Type II (open circles, dotted line) by day 7 and more frequent recovered placement than rats with severe spinal cord injury (SSCl) (B). Rats with $\mathrm{SSCl}$ showed forearm placement or Type $V$ (solid square, long dashed line) on day 3 and more frequently than in rats with $\mathrm{MSCI}(\mathbf{C})$. Type III (inverted solid triangle, short dashed line), Type IV (open triangle, dotted and dashed line). Error bars indicate standard error of the mean

contusion of SCI at the thoracic level to demonstrate transient behavioral deficits proceeding to near-complete recovery [11]. In the present study, the adapted weight-drop device mimics a situation such as an injury caused by a motor vehicle accident where the spinal vertebrae break and exert pressure on a part the of spinal cord generating a force that will cause a contusion injury to the cord.

The efficacy of mild hemicontusion of SCI in the cervical level was evaluated using sensory and motor functional deficits. The adhesive sticker removal task by rats with either MSCI and SSCI at day 14 after injury showed a removal time similar to that by rats in the SH group, and indicates sensory recovery within 14 days.

The horizontal ladder test had been used to evaluate skilled locomotor function as previously reported after a study of the contributions of supraspinal motor pathways on forelimb function following cervical hemicontusion [9]. In the present study, the error scored, the percentage of total rungs used, and forelimb contact, which indicated a dysfunctional forelimb, showed the characteristics of graded injury. Rats with SSCI showed a greater severity of motor dysfunction with an increase in error scores and the percentage of total rungs used, and decrease in the percentage of correct paw placement. Normal recovered placement of paw on rungs (Type II) was observed 

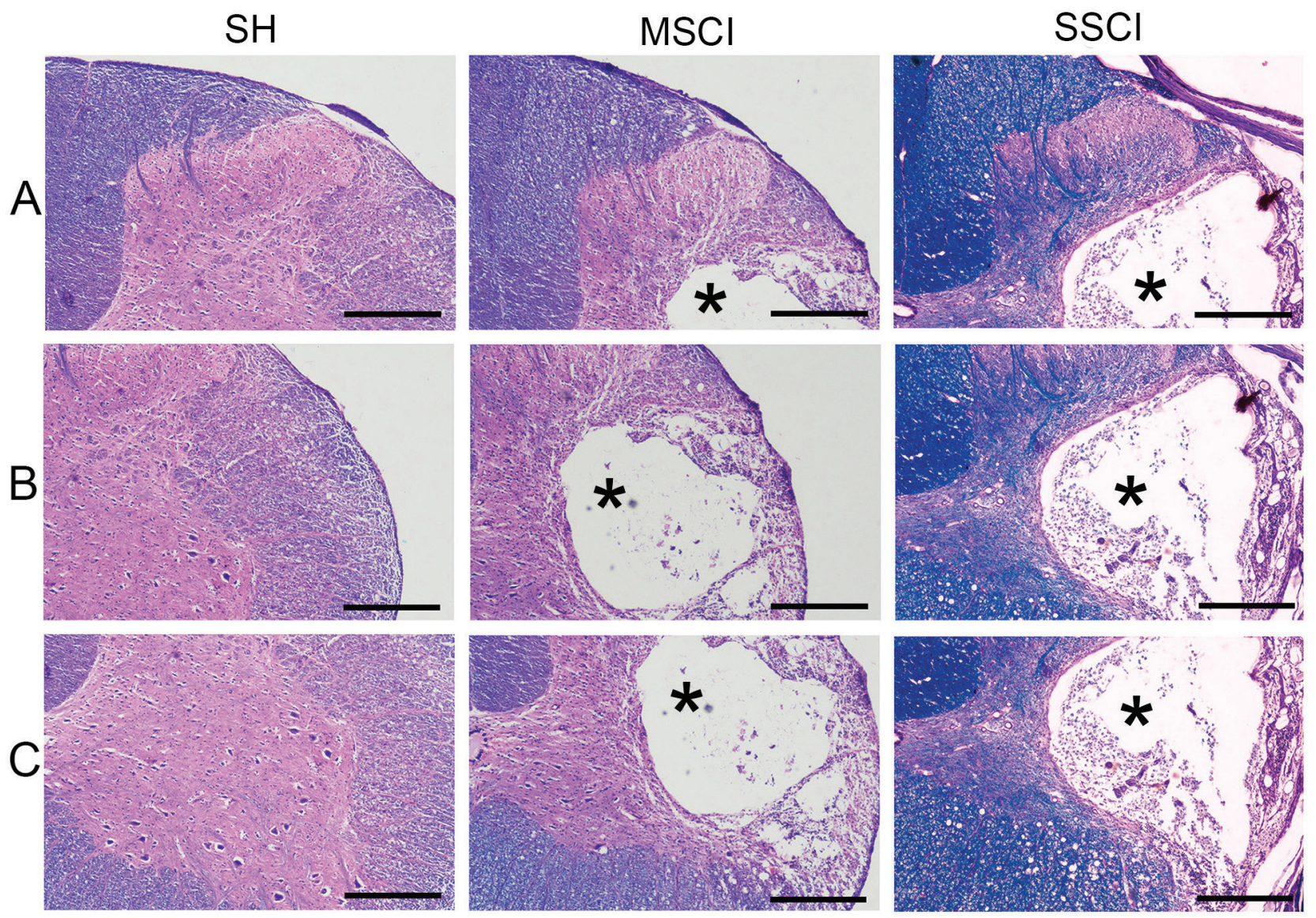

Figure 7. Comparison of histopathological changes demonstrated by Luxol Fast Blue counterstained with hematoxylin and eosin in the rats with sham (laminectomy only) injury (SH), mild spinal cord injury ( $\mathrm{MSCl}$ ), and severe $\mathrm{SCl}(\mathrm{SSCl})$. The transverse sections of the spinal cord at $\mathrm{C5}$ showing the dorsal horn (A), white matter (B), and ventral horn (C). MSCl and SSCI showing an intact dura mater and external surface the spinal tissue. The cystic cavity (asterisks) in rats with $\mathrm{SSCl}$ was larger than that in rats with $\mathrm{MSCl}$. Scale bars represent $400 \mu \mathrm{m}$

on day 7 after injury, and recovery was faster for rats with MSCI than it was for rats with SSCI. Histopathology indicated the size and site of lesion (indicated by the cystic cavity) in rats with either MSCI or SSCI could be localized with specificity that did not involve the dorsal column of the spinal cord. The lesion site in the rats with MSCI showed the cystic cavity in the dorsolateral funiculus. Onifer et al. reported that the dorsolateral funiculi do not transmit forepaw sensory information [12]. They also suggested that dorsolateral funiculus motor tract injuries do not interfere with the rat's ability to remove the adhesive stickers [12]. Therefore, the somatosensory sensation was affected only for about the first week after the injury caused by the surgical procedures including the laminectomy and all groups showed complete recovery in 14 days.

After injury, rats showed a clubbing forepaw on their ipsilateral side and normal forepaw at contralateral side. McKenna et al. [13] suggested that an injury at the level of the C5 vertebra causes damage to descending inputs to motor neuron pools that supply digital flexors of the ipsilateral forepaw. The lesion was unilateral; therefore, the contralateral forepaw was assessed as an internal normal control. In SSCI the greater force of the weight drop would produce larger lesion which involved the dorsal longitudinal fasciculus, ventrolateral funiculus, and most of the gray matter. By contrast, a smaller lesion size involving more specific tracts would be more useful because it would allow rats to perform better in specific behavioral tasks. To evaluate motor dysfunction in rats with SCI that were unable to remove a sticker, only the latency of initial contact to sense the stimulus can be used rather than the time to remove the sticker.

In the rats with MSCI group, the cystic cavity located in the dorsolateral funiculus involved the corticospinal and rubrospinal tract. The corticospinal and rubrospinal tracts have been implicated in initiating and modulating voluntary and goal-directed skilled movements [3,14]. Dunham et al. reported that the horizontal ladder test requires 3 sequences of movement: (1) aiming of the limb to the rung, this sequence requires proximal muscle contraction to swing the limb to 
the appropriate rung [9]. The proximal muscle contraction is supplied by vestibulospinal and reticulospinal pathways that are located at ventromedial funiculi [15]. (2) Placement of the paw on the rung, requiring tactile stimulation [9]. The fasciculus cuneatus located in the dorsal column is associated with tactile sensation response, injuries to this area may contribute to loss of placing response [12]. (3) Grasping the rung is associated with the coordination between paw flexors and extensors [9]. These muscles were supplied by rubrospinal and corticospinal tracts, and the motor neuron pools at the level of the C5 vertebra that are correlated with skilled and goal movement, and grip strength $[15,16]$. Using a modified tip with a small diameter in the adapted weight-drop device may contribute to MSCI for studies of recovery because the lesion site and size was appropriate for specific evaluation of the histopathological and functional recovery from SCI. A limitation of the small tip is that it may cause only partial functional deficit that is difficult to evaluate in smaller or larger experimental animals. Therefore, tip size should be optimized for the size of the animals before the weight-drop device is used for experiments.

A limitation of the present technique is that the results are highly variable. The error bars that represent SEM are large, especially in Figures 5E, and $\mathbf{7 B}$ and $\mathbf{C}$. In general, the present model produces a high variability of results as found by others [17-24]. Other limitations include that we have not used computer control of the impactor because of our limited resources [17, 23] and we have not measured evoked potentials as others have done [23].

\section{Conclusions}

The adapted weight-drop device is suitable for creating a rat model of SCI. The device is simple, reliable, and consistently produces a mild degree of injury. Mild deficits may be useful for evaluating therapeutic interventions for SCI.

Author contributions. KT, ST, and SC contributed substantially to the conception and design of this study. $\mathrm{KB}, \mathrm{AV}$, and NT contributed substantially to the acquisition of data. KT, ST, and SC analyzed and interpreted the data. All the authors contributed to drafting and critical revision of the manuscript, approved the final version submitted, and take responsibility for statements made in the published article.

Acknowledgments. The authors thank Mr. Wuttinan Theerathananon, Department of Physiology, for his advice and assistance in the development of methods and are grateful to Mrs. Natchaya Amornmettajit, Department of Anatomy, for her assistance in the histological processing. The adapted weight device was made and adjusted by Mr. Pinit Choorod. We did not receive any specific grant for this research from any funding agency in the public, commercial or not-for-profit sectors. The present work was presented in part as a poster at the 41st Annual Conference of the Anatomy Association of Thailand (AAT41), 2018 May 23-25, Cha-am, Phetchaburi, Thailand. Kanyaratana Bamrungsuk, Kanokwan Tilokskulchai, Sompol Tapechum, Supin Chompoopong. Behavioral and histopathological studies of mild cervical spinal cord contusion injury using adopted weight-drop device in the rat [PP 04, AAT149].

Conflict of interest statement. The authors have each completed and submitted an International Committee of Medical Journal Editors Uniform Disclosure Form for Potential Conflicts of Interest. None of the authors disclose any conflict of interest.

\section{References}

[1] Muir GD, Webb AA. Mini-review: assessment of behavioural recovery following spinal cord injury in rats. Eur J Neurosci. 2000; 12:3079-86.

[2] Pearse DD, Lo TP Jr, Cho KS, Lynch MP, Garg MS, Marcillo AE, et al. Histopathological and behavioral characterization of a novel cervical spinal cord displacement contusion injury in the rat. J Neurotrauma. 2005; 22:680-702.

[3] Soblosky JS, Song JH, Dinh DH. Graded unilateral cervical spinal cord injury in the rat: evaluation of forelimb recovery and histological effects. Behav Brain Res. 2001; 119:1-13.

[4] Tan AM, Petruska JC, Mendell LM, Levine JM. Sensory afferents regenerated into dorsal columns after spinal cord injury remain in a chronic pathophysiological state. Exp Neurol. 2007; 206:257-68.

[5] Grow JL, Liu YQ, Barks JD. Can lateralizing sensorimotor deficits be identified after neonatal cerebral hypoxia-ischemia in rats? Dev Neurosci. 2003; 25:394-402.

[6] Takahashi Y, Nakajima Y. Dermatomes in the rat limbs as determined by antidromic stimulation of sensory C-fibers in spinal nerves. Pain. 1996; 67:197-202.

[7] Zhang Y, Xing S, Zhang J, Li J, Li C, Pei Z, Zeng J. Reduction of $\beta$-amyloid deposits by $\gamma$-secretase inhibitor is associated with the attenuation of secondary damage in the ipsilateral thalamus and sensory functional improvement after focal cortical infarction in hypertensive rats. J Cereb Blood Flow Metab. 2011; 31:572-9.

[8] Muir GD, Webb AA, Kanagal S, Taylor L. Dorsolateral cervical spinal injury differentially affects forelimb and hindlimb action in rats. Eur J Neurosci. 2007; 25:1501-10.

[9] Dunham KA, Siriphorn A, Chompoopong S, Floyd CL. Characterization of a graded cervical hemicontusion spinal cord injury model in adult male rats. J Neurotrauma. 2010; 27:2091-106.

[10] Sribnick EA, Samantaray S, Das A, Smith J, Matzelle DD, Ray SK, Banik NL. Post-injury estrogen treatment of chronic spinal cord 


\section{Allli ASIAN BIOMEDICINE}

injury improves locomotor function in rats. J Neurosci Res. 2010; 88:1738-50.

[11] Scheff S, Rabchevsky A, Fugaccia I, Main J, Lumpp JE Jr. Experimental modeling of spinal cord injury: characterization of a force-defined injury device. J. Neurotrauma. 2003; 20:179-93.

[12] Onifer SM, Zhang YP, Burke DA, Brooks DL, Decker JA, McClure NJ, et al. Adult rat forelimb dysfunction after dorsal cervical spinal cord injury. Exp Neurol. 2005; 192:25-38.

[13] McKenna JE, Prusky GT, Whishaw IQ. Cervical motoneuron topography reflects the proximodistal organization of muscles and movements of the rat forelimb: a retrograde carbocyanine dye analysis. J Comp Neurol. 2000; 419:286-96.

[14] Gensel JC, Tovar CA, Hamers FP, Deibert RJ, Beattie MS, Bresnahan JC. Behavioral and histological characterization of unilateral cervical spinal cord contusion injury in rats. J Neurotrauma. 2006; 23:36-54.

[15] Anderson KD, Gunawan A, Steward O. Quantitative assessment of forelimb motor function after cervical spinal cord injury in rats: relationship to the corticospinal tract. Exp Neurol. 2005; 194:161-74.

[16] Metz GA, Whishaw IQ. Cortical and subcortical lesions impair skilled walking in the ladder rung walking test: a new task to evaluate fore- and hindlimb stepping, placing, and co-ordination. J Neurosci Methods. 2002; 115:169-79.
[17] Krishna V, Andrews H, Jin X, Yu J, Varma A, Wen X. A contusion model of severe spinal cord injury in rats. JoVE. 2013;78: 50111. doi: 10.3791/50111

[18] Panjabi MM, Wrathall JR. Biomechanical analysis of experimental spinal cord injury and functional loss. Spine 1988; 13:1365-1370.

[19] Geissler SA, Schmidt CE, Schallert T. Rodent models and behavioral outcomes of cervical spinal cord injury. J Spine. 2013; Suppl 4: 001. doi: 10.4172/2165-7939.S4-001

[20] Khan T, Havey RM, Sayers ST, Patwardhan A, King WW. Animal models of spinal cord contusion injuries. Lab Anim Sci. 1999; 49:161-72.

[21] Nardone R, Florea C, Höller Y, Brigo F, Versace V, Lochner P, Golaszewski S, Trinka E. Rodent, large animal and non-human primate models of spinal cord injury. Zoology (Jena). 2017; 123:101-14.

[22] Kwon BK, Hillyer J, Tetzlaff W. Translational research in spinal cord injury: a survey of opinion from the SCI community. J. Neurotrauma. 2010; 27:21-33.

[23] Onifer SM, Rabchevsky AG, Scheff SW. Rat models of traumatic spinal cord injury to assess motor recovery. ILAR J. 2007; 48:385-95.

[24] Beattie MS, Bresnahan JC. Cell death, repair, and recovery of function after spinal cord contusion injuries in rats. In: Kalb RG, Strittmatter SM, editors. Neurobiology of spinal cord injury. Humana Press; Totowa, NJ: 2000. pp. 1-21. 